\begin{tabular}{|c|l|}
\hline Title & A level set approach to semicontinuous viscosity solutions for Cauchy problems \\
\hline Author(s) & Giga, Y.; Sato, M.-H \\
\hline Citation & Hokkaido University Preprint Series in Mathematics, 472, 1-32 \\
\hline Issue Date & 1999-9-1 \\
\hline DOI & 10.14943/83618 \\
\hline Doc URL & http://hdl.handle.net/2115/69222 \\
\hline Type & bulletin (article) \\
\hline File Information & pre472.pdf \\
\hline
\end{tabular}

Instructions for use 
A LEVEL SET APPROACH TO SEMICONTINUOUS VISCOSITY SOLUTIONS FOR CAUCHY PROBLEMS

Yoshikazu Giga and Moto-Hiko Sato

Series \# 472. September 1999 


\title{
A LEVEL SET APPROACH TO SEMICONTINUOUS VISCOSITY SOLUTIONS FOR CAUCHY PROBLEMS
}

\author{
Yoshikazu Giga and Moto-Hiko Sato \\ Department of Mathematics \\ Hokkaido University \\ Sapporo 060-0810, Japan \\ Muroran Institute of Technology \\ 27-1 Mizumoto, Muroran 050-8585 \\ Japan
}

\begin{abstract}
A new notion of solution (called $L$-solution) is introduced so that the Cauchy problem for the Hamilton-Jacobi equation with semicontinuous initial data is globally solvable. No convexity assumptions on Hamiltonians are imposed. The solution is interpreted as the level set of an auxiliary problem. It turns out that our $L$ solution is consistent with a classical discontinuous viscosity solution and a bitateral viscosity solution. Moreover, our $L$-solution is unique and enjoy the comparison principle. The condition that initial data is really attained is also discussed.
\end{abstract}

\section{Introduction}

We are concerned with semicontinuous viscosity solutions of the Cauchy problem for the Hamilton-Jacobi equations or more general degenerate parabolic equations. Our main goal is to find a suitable notion of a solution when initial data is discontinuous.

The idea of viscosity solution was first introduced by M. G. Crandall and P.-L. Lions [CL] to first order fully nonlinear equations. A viscosity solution is a kind of 
weak solution which is not based on variational structure but on order-preserving structure of the equations. The theory of viscosity solutions yields the unique existence of a global continuous (not necessarily differentiable) solution of the Cauchy problem for various kind of first and second order degenerate parabolic equations when initial data is at least continuous. For the development of the basic theory we refer to $[\mathrm{L}],[\mathrm{B} 2]$ for the first order equations and [CIL] for the second order equations. For example, it is well-known [CL], [L] that the initial value problem for the Hamilton-Jacobi equation of form

$$
\begin{aligned}
& u_{t}+H\left(x, u_{x}\right)=0 \quad \text { in }(0, T) \times \mathbf{R}^{n} \\
& u(0, x)=u_{0}(x) \quad x \in \mathbf{R}^{n}
\end{aligned}
$$

admits a unique global (uniformly) continuous viscosity solution when $u_{0}$ is bounded, uniformly continuous and $H$ is enough regular; for example,

$$
\begin{aligned}
& |H(x, p)-H(x, q)| \leq C|p-q| \\
& |H(x, p)-H(y, p)| \leq C(1+|p|)|x-y|
\end{aligned}
$$

with $C>0$ independent of $x, y, p, q \in \mathbf{R}^{n}$; see also [I1]. Here $u_{t}=\partial u / \partial t, u_{x}=$ $\left(\partial u / \partial x_{1}, \ldots, \partial u / \partial x_{n}\right)$ and $T>0$. The notion of viscosity solution has been extended to semicontinuous functions rather than continuous functions. This extension is important to prove the existence of solutions without appealing hard estimates. Such a method was first introduced by $\mathrm{H}$. Ishii [I2] which is regarded as an extension of Perron's method for the Laplace equation. However, if $u_{0}$ is, for example, merely upper semicontinuous, it is not clear that a classical semicontinuous viscosity solution is unique. For example, it is known [BSS] that solution of

$$
u_{t}+(x-t)\left|u_{x}\right|=0, \quad x \in \mathbf{R}, t>0
$$

starting with a characteristic function of some closed interval is not unique.

In [BJ1] E. N. Barron and R. Jensen introduced another notion of viscosity solutions for semicontinuous functions when the Hamiltonian $H=H(x, p)$ is concave 
in $p$ and proved the existence and the uniqueness of their solution for (1.1a), (1.1b) for bounded (from above), upper semicontinuous initial data $u_{0}$. Their solution is called a bilateral viscosity solution in $[\mathrm{BC}]$ and the idea has been extended in various settings by $\mathrm{G}$. Barles $[\mathrm{B} 1]$ and $\mathrm{S}$. Koike $[\mathrm{K}]$. The theory applies to optimal control problems [BJ2]; however, their theory is limited for concave $H$. (In [BJ1] $H$ is assumed to be convex but they consider the terminal value problem which is easily transformed to the initial value problem with concave Hamiltonian by setting $T-t$ by $t$.) As explained in [BC] (and in Bardi's article of [BCESS]) there are several other approaches to handle viscosity solutions with discontinuous data like envelope solutions. However, definition of such a solution contains sup or inf operation of a family of solutions or limiting procedure. So it is not directly checkable whether a function is such a solution. One of advantages of bilateral solutions is that its definition is direct without containing sup or inf operation of a family of solutions nor limiting procedure.

In this paper we introduce a new notion of a solution which does not contain sup or inf operation of a family of solutions nor limiting procedure for the Cauchy problem with upper semicontinuous initial data. Our notion does not require convexity of $H(x, p)$ in $p$ when we consider (1.1a). We call our solution an $L$-solution since it reflects the idea of the level set method ([CGG1], [ESp], [BCESS], [G]) of surface evolution equations. We shall prove the unique existence of solutions for various initial value problems and compare with other notion of solutions. For simplicity we explain our $L$-solution for (1.1a), (1.1b). We consider an auxiliary problem

$$
\begin{aligned}
& \psi_{t}-\psi_{y} H\left(x,-\psi_{x} / \psi_{y}\right)=0 \quad \text { in }(0, T) \times \mathbf{R}^{n+1} \\
& \psi(0, x, y)=\psi_{0}(x, y), \quad(x, y) \in \mathbf{R}^{n} \times \mathbf{R} .
\end{aligned}
$$

The equation (1.3a) is called the level set equation for the evolution of the graph of (1.1a). In fact, if a level set of a solution of $\psi$ of (1.3a) is given as the graph of a function $u(t, x)$, then $u$ must solve (1.1a). (The equation (1.3a) has already been proposed by A. I. Subbotin [S, Chapter I, Section 4.2] for a different purpose.) Let $u_{0}$ be an upper semicontinuous function on $\mathbf{R}^{n}$ with values in $\mathbf{R} \cup\{-\infty,+\infty\}$, i.e., 
$u_{0} \in \operatorname{USC}\left(\mathbf{R}^{n}\right)$. We define $\psi_{0}$ by

$$
\psi_{0}(x, y)=-\min \left\{\operatorname{dist}\left((x, y), K_{0}\right), 1\right\}
$$

with

$$
K_{0}=\left\{(x, y) \in \mathbf{R}^{n} \times \mathbf{R} ; y \leq u_{0}(x)\right\}
$$

We solve $(1.3 \mathrm{a}),(1.3 \mathrm{~b})$ with initial data $\psi_{0}$ defined in $(1.4 \mathrm{a}),(1.4 \mathrm{~b})$. We define our $L$-solution by

$$
\bar{u}(t, x)=\sup \{y \in \mathbf{R} ; \psi(t, x, y) \geq 0\}
$$

where $\psi$ is the continuous viscosity solution of $(1.3 \mathrm{a}),(1.3 \mathrm{~b})$. The solution $\psi$ uniquely exists globally in time under suitable assumptions on $H$. For example, if $(1.2 \mathrm{a}),(1.2 \mathrm{~b})$ is fulfilled, and the recession function

$$
H_{\infty}(x, p)=\lim _{\lambda \downarrow 0} \lambda H(x, p / \lambda), \quad x \in \mathbf{R}^{n}, p \in \mathbf{R}^{n}
$$

exists, then the singularity at $\psi_{y}=0$ in (1.3a) is removable if we restrict $\psi$ satisfying $\psi_{y} \leq 0$. It turns out that the monotonicity of $\psi$ in $y$ is preserved for $t>0$, so under the above assumptions on $H(1.3 \mathrm{a}),(1.3 \mathrm{~b})$ admits a unique uniformly continuous solution $\psi=\psi(t, x, y)$ which is nonincreasing in $y$ for a given $\psi_{0}$ defined by (1.4a), $(1.4 \mathrm{~b})$.

We also notice that one may replace $\psi_{0}$ by any other uniformly continuous function $\tilde{\psi}_{0}$ which is nonincreasing in $y$ and $K_{0}=\left\{(x, y) \in \mathbf{R}^{n} \times \mathbf{R} ; \tilde{\psi}_{0}(x, y) \leq 0\right\}$. This follows from well-known properties of the level set equation as uniqueness of the level set of solutions ([CGG1], [ESp], [G], [IS]). If $u_{0}$ is uniformly continuous, this guarantees that one may take $\psi_{0}(x, y)=u_{0}(x)-y$. This observation shows that our $L$-solution $\bar{u}$ agrees with classical viscosity solution $u$ when $u_{0}$ is uniformly continuous, if we notice that $\psi(t, x, y)=u(t, x)-y$.

We shall also prove that an $L$-solution $\bar{u}$ is a viscosity solution even if $\bar{u}$ is not continuous (Theorem 3.7 (i)). For this purpose we recall a variant of the slicing lemma in [GG] and prove that $\bar{u}^{*}$ is a subsolution. To prove that $\bar{u}_{*}$ is a supersolution we 
need the monotonicity property of $\psi$ in $y$ as well as the slicing lemma. Here $u^{*}$ denotes the upper semicontinuous envelope while $u_{*}$ is the lower semicontinuous envelope.

When $H(x, p)$ is convex in $p$, it turns out (Theorem 3.8) that an $L$-solution of (1.1a), (1.1b) agrees with the bilateral viscosity solution in [BJ1]. To show this fact we notice the property that an $L$-solution and a bilateral viscosity solution are given as a monotone decreasing limit of continuous viscosity solutions (Lemma 4.4).

It turns out that an L-solution of (1.1a), (1.1b) is given as the supremum of the family of all subsolution $v$ such that $v(0, x) \leq u_{0}(x)$ at least when $H$ satisfies (1.2a), (1.2b) and $\sup _{x, p}|H(x, p)| /|p|<\infty$ as well as the existence of $H_{\infty}$. In other words, the $L$-solution is a maximal subsolution which is initially less than $u_{0}$ (Theorem 5.1). Thus our $L$-solution is closely related to envelope solution defined for the Dirichlet problem for infinite horizon problem as explained in [BC]; see also [S].

It is not clear in what sense the initial value is attained for $L$-solution (unless $\bar{u}$ is continuous up to $t=0$.) Since the set $\{\psi \geq 0\}$ is closed in $[0, T) \times \mathbf{R}^{n} \times \mathbf{R}, \bar{u}$ is upper semicontinuous at $t=0$, i.e.,

$$
u_{0}(x) \geq \varlimsup_{\substack{t \downarrow j \rightarrow x \\ y \rightarrow x}} \bar{u}(t, y) .
$$

However, it is not always clear whether there exists a sequence $t_{m} \rightarrow 0, y_{m} \rightarrow 0$ such that

$$
u_{0}(x)=\lim _{m \rightarrow \infty} \bar{u}\left(t_{m}, y_{m}\right)
$$

As in [CGG2], we say that $u$ is right accessible at $t=0$ if for each $x \in \mathbf{R}^{n}$ there exists $t_{m} \rightarrow 0, x_{m} \rightarrow x$ that satisfies (1.5) with $\bar{u}=u$. Here is an example that $\bar{u}$ is not right accessible at $t=0$. We consider

$$
u_{t}+\left|u_{x}\right|=0 \quad \text { in }(0, T) \times \mathbf{R}^{n}
$$

with $u(0, x)=0$ at $x=0, u(0, x)=-\infty$ for $x \neq 0$. Then our $L$-solution $\bar{u}(t, x) \equiv-\infty$ for $t>0$ so $\bar{u}$ is not right accessible (Corollary 6.4). We shall give criteria of right accessibility when $H$ depends only on $u_{x}$ in this paper (Theorems 6.2, 6.7).

Although we mainly restrict ourselves for first order equations with timedependent Hamiltonians, many assertions are still valid for second order equations. We mention these points at the end of related sections. 
Main results of this paper have been announced in [GS]. After this work was completed, we were informed of a recent work of M. Bardi related to ours. He developed the theory of envelope solutions for the Cauchy problem for general parabolic equations. He proved, among other results, that the maximal subsolution $u$ is an infimum of all lower semicontinuous supersolution which is initially greater than $u(0, x)$ where $u(0, x)$ is bounded and upper semicontinuous. Such a property is implicitly proved in our paper, since our L-solution is the maximal subsolution, approximated from above by continuous supersolution.

\section{Level set equations}

We consider the Hamilton-Jacobi equation of form

$$
u_{t}+H\left(t, x, u, u_{x}\right)=0
$$

where $u=u(t, x)$ is a real-valued function of $t \in(0, T), T>0$ and $x=\left(x_{1}, \cdots, x_{n}\right) \in$ $\mathbf{R}^{n} ; u_{x}$ denotes the gradient in the spatial variables $x$, i.e., $u_{x}=\left(\partial u / \partial x_{1}, \cdots\right.$, $\partial u / \partial x_{n}$ ) and $u_{t}=\partial u / \partial t$. (This is more general than (1.1a) since it allows timedependence in $H$.) Suppose that the graph of $u$ is given by the zero level set of an auxiliary function $\psi=\psi(t, x, y)$ defined on $(0, T) \times \mathbf{R}^{n} \times \mathbf{R}$ and that $\psi_{y}=\partial \psi / \partial y \neq 0$ on the zero level set of $\psi$. Differentiating $\psi(t, x, u(t, x))=0$ in $t$ and $x$ we get $\psi_{t}+\psi_{y} u_{t}=0, \psi_{x}+\psi_{y} u_{x}=0$. We thus observe that (2.1) is formally equivalent to

$$
\psi_{t}-\psi_{y} H\left(t, x, y,-\psi_{x} / \psi_{y}\right)=0
$$

on the zero level set of $\psi$, i.e., $\{\psi=0\}$. We consider (2.2) not only on $\{\psi=0\}$ but also in whole $(0, T) \times \mathbf{R}^{n} \times \mathbf{R}$ and say that (2.2) is the level set equation of (the evolution law of the graph of $u$ given by) (2.1).

We first recall comparison principle for

$$
\psi_{t}+F\left(t, z, \psi, \psi_{z}\right)=0 \quad \text { in } \quad(0, T) \times \mathbf{R}^{N}
$$


which is contained in [I1] and [GGIS]. We list a standard set of assumptions on $F$.

(F1) $F$ is continuous in $Z=[0, T] \times \mathbf{R}^{N} \times \mathbf{R} \times \mathbf{R}^{N}$, i.e., $F \in C(Z)$.

(F2) There exists a modulus $m_{1}$ (i.e., $m_{1} \in C[0, \infty)$ is nondecreasing and $m_{1}(0)=0$ ) such that

$$
\left|F(t, z, r, \xi)-F\left(t, z^{\prime}, r, \xi\right)\right| \leq m_{1}\left(\left|z-z^{\prime}\right|(|\xi|+1)\right)
$$

for all $(t, z, r, \xi) \in Z$ and $z^{\prime} \in \mathbf{R}^{N}$.

(F3) For each $C_{1}>0$ there exists a modulus $m_{2}$ such that

$$
\left|F(t, z, r, \xi)-F\left(t, z, r, \xi^{\prime}\right)\right| \leq m_{2}\left(\left|\xi-\xi^{\prime}\right|\right)
$$

for all $(t, z, r, \xi) \in Z, \xi^{\prime} \in \mathbf{R}^{N}$ satisfying $|\xi|,\left|\xi^{\prime}\right| \leq C_{1}$.

(F4) $r \mapsto F(t, z, r, \xi)$ is nondecreasing.

Theorem 2.1 (Comaprison principle). Assume that $F$ satisfies (F1)-(F4). Let $\psi_{1}$ and $\psi_{2}$ be, respectively, viscosity sub- and supersolutions of $(2.3)$ in $(0, T) \times \mathbf{R}^{N}$. Assume that

$$
\sup _{t \in[0, T)} \sup _{z \in \mathbb{R}^{N}} \psi_{1}(t, z) /(1+|z|)<\infty, \inf _{t \in[0, T)} \inf _{z \in \mathbb{R}^{N}} \psi_{2}(t, z) /(1+|z|)>-\infty
$$

If

$$
\lim _{\delta \downarrow 0} \sup \left\{\psi_{1}^{*}(t, z)-\psi_{2 *}\left(s, z^{\prime}\right) ; 0 \leq t, s \leq \delta,\left|z-z^{\prime}\right| \leq \delta\right\} \leq 0,
$$

then there exists modulus $m$ such that

$$
\psi_{1}^{*}(t, z)-\psi_{2 *}\left(t, z^{\prime}\right) \leq m\left(\left|z-z^{\prime}\right|\right)
$$

for $t \in(0, T), z, z^{\prime} \in \mathbf{R}^{N}$. In particular $\psi_{1}^{*} \leq \psi_{2 *}$ on $[0, T) \times \mathbf{R}^{N}$.

Here $\psi^{*}$ denotes the upper semicontinuous envelope of $\psi$ and $\psi_{*}=-(-\psi)^{*}$. The condition (2.4) is fulfilled if $\psi_{1}(0, z) \leq \psi_{2}(0, z), z \in \mathbf{R}^{N}$ provided that $\psi_{1}$ and $\psi_{2}$ are uniformly continuous on $\left[0, T^{\prime}\right) \times \mathbf{R}^{N}$ for some $T^{\prime} \in(0, T)$. The next existence result is contained in [I1]. 
Theorem 2.2 (Existence). Assume that $F$ satisfies (F1)-(F4). Assume that $\psi_{0}$ is uniformly continuous in $\mathbf{R}^{N}$ (shortly $\psi_{0} \in U C\left(\mathbf{R}^{N}\right)$ ). Then there is a unique viscosity solution $\psi$ of (2.3) with $\left.\psi\right|_{t=0}=\psi_{0}$ such that $\psi$ is uniformly continuous $\left[0, T^{\prime}\right] \times \mathbf{R}^{N}$ for each $T^{\prime} \in(0, T)$, (so that

$$
\left.\sup _{t \in\left[0, T^{\prime}\right]} \sup _{x \in \mathbf{R}^{N}}|\psi(t, x)| /(|x|+1)<\infty .\right)
$$

If $\psi_{0}$ is bounded, then $\psi$ is bounded in $(0, T) \times \mathbf{R}^{N}$.

The uniqueness follows from Theorem 2.1 since such solutions $\psi_{1}$ and $\psi_{2}$ satisfy (2.4) and the growth condition in Theorem 2.1 if $\psi_{1} \leq \psi_{2}$ at $t=0$.

We apply these theorems for $(2.2)$ by setting

$$
F(t, z, \xi)=F(t, x, y, p, q)= \begin{cases}-q H(t, x, y,-p / q) & q<0 \\ H_{\infty}(t, x, y, p) & q \geq 0\end{cases}
$$

where $H_{\infty}$ is the recession function:

$$
H_{\infty}(t, x, y, p)=\lim _{\lambda \downarrow 0} \lambda H(t, x, y, p / \lambda), x \in \mathbf{R}^{n}, y \in \mathbf{R}, p \in \mathbf{R}^{n} .
$$

Here $z=(x, y) \in \mathbf{R}^{n} \times \mathbf{R}=\mathbf{R}^{N}$ and $\xi=(p, q) \in \mathbf{R}^{n} \times \mathbf{R}=\mathbf{R}^{N}$. Since $F$ in (2.6) is independent of $r,(\mathrm{~F} 4)$ is automatically fulfilled. We shall rewrite conditions for $H$ so that $F$ defined by (2.6) fulfills (F1)-(F3).

(H1) $H \in C\left([0, T] \times \mathbf{R}^{n} \times \mathbf{R} \times \mathbf{R}^{n}\right)$ and $H_{\infty}(t, x, y, p)$ exists for $x \in \mathbf{R}^{n}, y \in \mathbf{R}$, $p \in \mathbf{R}^{n}, t \in[0, T]$.

(H2) There exists a modulus $m_{1}$ such that

$$
\left|q H(t, x, y,-p / q)-q H\left(t, x^{\prime}, y^{\prime},-p / q\right)\right| \leq m_{1}\left(\left(\left|x-x^{\prime}\right|+\left|y-y^{\prime}\right|\right)(|p|+|q|+1)\right)
$$

for all $x, x^{\prime} \in \mathbf{R}^{n}, y, y^{\prime} \in \mathbf{R}, p \in \mathbf{R}^{n}, q<0, t \in[0, T]$.

(H3) For each $C_{1}>0$ there exists a modulus $m_{2}$ such that

$$
\left|q H(t, x, y,-p / q)-q H\left(t, x, y,-p^{\prime} / q^{\prime}\right)\right| \leq m_{2}\left(\left|p-p^{\prime}\right|+\left|q-q^{\prime}\right|\right)
$$


for all $x \in \mathbf{R}^{n}, y \in \mathbf{R}, t \in[0, T], p, p^{\prime} \in \mathbf{R}^{n}, q, q^{\prime}<0$ satisfying $|p|,\left|p^{\prime}\right|,|q|,\left|q^{\prime}\right| \leq C_{1}$. Note that condition (H1)-(H3) is weaker than (1.2a), (1.2b) provided that $H_{\infty}$ exists.

Lemma 2.3. The condition (H1)-(H3) is equivalent to condition (F1)-(F3) if $F$ is of form (2.6).

Clearly, the condition (F1)-(F3) implies (H1)-(H3). By the existence of $H_{\infty}$ in (H1) it is easy to derive (F2)-(F3) from (H2)-(H3). Since $H$ is continuous, $F$ is continuous for $q<0$. The continuity $q=0$ follows from (H2), (H3) and the existence of $H_{\infty}$.

Example 2.4. (i) $H(p)=\left(1+|p|^{2}\right)^{1 / 2}$ so that $F(p, q)=\left(q^{2}+|p|^{2}\right)^{1 / 2}$ for $q<0$, $F(p, q)=|p|$ for $q \geq 0$. By definition (H1) and (H2) are fulfilled. Since $F$ is globally Lipschitz, (H3) follows.

(ii) $H(p)=|p|^{\alpha}, 0<\alpha \leq 1$ so that $F(p, q)=|q|^{1-\alpha}|p|^{\alpha}$ for $q<0, F(p, q)=0$ for $q \geq$ 0 . The condition (H1), (H2) is trivially fulfilled. Since $F$ is locally Hölder continuous, (H3) follows. This example does not satisfy (1.2a) so (H1)-(H3) is actually weaker than (1.2a), (1.2b) provided that $H_{\infty}$ exists.

(iii) $H(t, x, p)=a(x, t)|p|$ satisfies (H1)-(H3) provided that $a$ is continuous in $\mathbf{R}^{N} \times$ $[0, T]$ and that $a(x, t)$ is Lipschitz in $x \in \mathbf{R}^{N}$ uniformly in $t \in[0, T]$. Thus the equation $u_{t}+(x-t)\left|u_{x}\right|=0$ in the Introduction is of form (2.1) satisfying (H1)-(H3).

Remark 2.5. The procedure to derive (2.2) from (2.1) can be easily extended even if (2.1) is replaced by a general second-order equation:

$$
u_{t}+G\left(t, x, u, u_{x}, u_{x x}\right)=0
$$

where $u_{x x}$ denotes the Hessian matrix of $u$. Differentiating $\psi(t, x, u(t, x))=0$ twice in $x$ we get

$$
\psi_{x x}+2 \psi_{x y} \otimes u_{x}+\psi_{y y} u_{x} \otimes u_{x}+\psi_{y} u_{x x}=0
$$

where $\otimes$ denotes the tensor product of $n$-vectors. Thus the 'level set equation' (corresponding to $(2.2))$ of $(2.7)$ is

$$
\psi_{t}-\psi_{y} G\left(t, x, y,-\psi_{x} / \psi_{y},-\left\{\psi_{x x}-2 \psi_{x y} \otimes \psi_{x} / \psi_{y}+\psi_{y y} \psi_{x} \otimes \psi_{x} / \psi_{y}^{2}\right\} / \psi_{y}\right)=0
$$


If (2.7) is the mean curvature flow equation for the graph of $u$, i.e.,

$$
u_{t}-\sqrt{1+\left|u_{x}\right|^{2}} \operatorname{div}_{x}\left(u_{x} / \sqrt{1+\left|u_{x}\right|^{2}}\right)=0
$$

it is easy to see that (2.8) is nothing but $\psi_{t}-|D \psi| \operatorname{Div}(D \psi /|D \psi|)=0$ which is the level set equation for the mean curvature flow equation in the level set method developed by [CGG1], [ESp]; see [G] for review. Here $D \psi=\left(\psi_{x}, \psi_{y}\right)$ and Div is the divergence in $\mathbf{R}^{n+1}$. This justifies the name of 'the level set equation'.

In general (2.8) has strong singularity at $\psi_{y}=0$. For example, if (2.7) is the heat equation, as observed in $[\mathrm{E}]$ the second term in (2.8) is singular as $\psi_{y} \rightarrow 0$ of order $1 / \psi_{y}^{2}$. So, it is not clear whether the comparison principle like Theorem 2.1 is still valid. Moreover, such an equation is difficult to solve for arbitrary initial data $\psi_{0}$ as pointed out in [E], [CGS].

\section{3. $L$-solutions}

We introduce a new notion of a solution called an $L$-solution for the initial value problem

$$
\begin{array}{ll}
u_{t}+H\left(t, x, u, u_{x}\right)=0 & \text { in } \quad(0, T) \times \mathbf{R}^{n} \\
u(0, x)=u_{0}(x), & x \in \mathbf{R}^{n},
\end{array}
$$

where $H(\cdot, p)$ is not necessarily concave in $p \in \mathbf{R}^{n}$.

\subsection{Construction of initial auxiliary function.}

Let $\operatorname{USC}(\Omega)$ be the space of all upper semicontinuous functions defined in $\Omega$ with values in $\mathbf{R} \cup\{-\infty,+\infty\}$, where $\Omega$ is a metric space. For $u_{0} \in \operatorname{USC}\left(\mathbf{R}^{n}\right)$ we set

$$
K_{0}=\left\{(x, y) \in \mathbf{R}^{n} \times \mathbf{R} ; y \leq u_{0}(x)\right\} .
$$


The set $K_{0}$ is closed since $u_{0}$ is upper semicontinuous. Thus if we set

$$
\psi_{0}(x, y)=-\min \left\{\operatorname{dist}\left((x, y), K_{0}\right), 1\right\}
$$

then

$$
\begin{aligned}
K_{0} & =\left\{(x, y) \in \mathbf{R}^{n} \times \mathbf{R} ; \psi_{0}(x, y) \geq 0\right\} \\
& =\left\{(x, y) \in \mathbf{R}^{n} \times \mathbf{R} ; \psi_{0}(x, y)=0\right\}
\end{aligned}
$$

Since $\left(x, y_{0}\right) \in K_{0}$ implies $(x, y) \in K_{0}$ for all $y \leq y_{0}$, we see that $y \mapsto \psi_{0}(x, y)$ is nonincreasing. Since $\psi_{0}$ is Lipschitz continuous, we have proved:

Proposition 3.1. For $K_{0}$ defined by (3.2) with $u_{0} \in U S C\left(\mathbf{R}^{n}\right)$ there exists a bounded, uniformly continuous function $\psi_{0}$ on $\mathbf{R}^{n} \times \mathbf{R}$ (shortly, $\psi_{0} \in B U C\left(\mathbf{R}^{n} \times \mathbf{R}\right)$ ) such that $y \mapsto \psi_{0}(x, y)$ is nonincreasing and $K_{0}=\left\{(x, y) \in \mathbf{R}^{n} \times \mathbf{R} ; \psi_{0}(x, y) \geq 0\right\}$.

\subsection{Monotonicity preserving}

We consider the initial value problem for the level set equation of (3.1a), i.e.

$$
\begin{aligned}
& \psi_{t}+F\left(t, x, y, \psi_{x}, \psi_{y}\right)=0 \quad \text { in } \quad(0, T) \times \mathbf{R}^{n} \times \mathbf{R} \\
& \psi(0, x, y)=\psi_{0}(x, y), \quad(x, y) \in \mathbf{R}^{n} \times \mathbf{R}
\end{aligned}
$$

where $F$ is defined by (2.6).

Lemma 3.2. Assume that $H$ satisfies (H1)-(H3). For $\psi_{0} \in U C\left(\mathbf{R}^{n}\right)$ there is a unique viscosity solution of (3.3a)-(3.3b) with $\psi \in U C\left(\left[0, T^{\prime}\right] \times \mathbf{R}^{n}\right)$ for and $T^{\prime} \in$ $(0, T)$. If $\psi_{0}$ is bounded, then $\psi$ is bounded on $(0, T) \times \mathbf{R}^{n}$. In addition to (H1)-(H3) assume that

(H4) $y \mapsto H(t, x, y, p)$ is nondecreasing.

If the function $y \mapsto \psi_{0}(x, y)$ is nonincreasing, then so is the function $y \mapsto \psi(t, x, y)$, $x \in \mathbf{R}^{n}, t \in(0, T)$ for all $x \in \mathbf{R}^{n}$. 
Proof. The unique existence of solution follows from Theorem 2.2 and Lemma 2.3.

For $\psi_{0}$ we set $\psi_{0}^{h}(x, y)=\psi_{0}(x, y+h)$ for $h>0$. Similarly for the solution $\psi$ we set

$$
\psi^{h}(t, x, y)=\psi(t, x, y+h)
$$

We observe that $\psi^{h}$ solves

$$
\partial_{t} \varphi+F\left(t, x, y+h, \varphi_{x}, \varphi_{y}\right)=0
$$

in the viscosity sense. By the monotonicity assumption (H4) we see $\psi^{h}$ solves

$$
\partial_{t} \varphi+F\left(t, x, y, \varphi_{x}, \varphi_{y}\right) \leq 0
$$

in the viscosity sense. Thus $\psi^{h}$ is a subsolution of (3.3a). Since $\psi_{0}^{h} \leq \psi_{0}$ and uniform continuity, by the comparison principle (Theorem 2.1) we have $\psi^{h} \leq \psi$ for all time. This implies that $y \mapsto \psi(t, x, y)$ is nonincreasing.

\subsection{Definition of $L$-solutions}

We are now in position to introduce a new notion of solutions for (3.1a)-(3.1b).

Definition 3.3. (i) Let $\bar{u} \in \operatorname{USC}\left(I \times \mathbf{R}^{n}\right)$ where $I$ is an open interval. We say that $\bar{u}$ is an $L$-solution (in $I \times \mathbf{R}^{n}$ ) if there is $\psi: I \times \mathbf{R}^{n} \times \mathbf{R} \rightarrow \mathbf{R}$ nonincreasing in the last variable $y$ that solves the level set equation (3.3a) of (3.1a) in $I \times \mathbf{R}^{n}$ in the viscosity sense with $\psi \in \mathrm{UC}\left(J \times \mathbf{R}^{n} \times \mathbf{R}\right)$ for any compact interval $J$ and that

$$
\bar{u}(t, x)=\sup \{y \in \mathbf{R} ; \psi(t, x, y) \geq 0\}, \quad t \in I, x \in \mathbf{R}^{n}
$$

We interpret that $\bar{u}(t, x)=-\infty$ if the set $\{y \in \mathbf{R} ; \psi(t, x, y) \geq 0\}$ is empty.

(ii) Assume that $I=(0, T)$ and that $u_{0} \in \mathrm{USC}\left(\mathbf{R}^{n}\right)$. We say that $\bar{u}$ is an $L$-solution of (3.1a)-(3.1b) if $\bar{u}$ is an $L$-solution in $(0, T) \times \mathbf{R}^{n}$ and moreover the function $\psi$ in (3.4) is uniformly continuous $\left[0, T^{\prime}\right] \times \mathbf{R}^{n} \times \mathbf{R}$ for any $T^{\prime} \in(0, T)$ such that $\left.\psi\right|_{t=0}=\psi_{0}$ as in Proposition 3.1. 
Since the solution $\bar{u}$ is defined via the level set equation, we call $\bar{u}$ an $L$-solution. For a given initial data $u_{0}$ there is a huge freedom to choose $\psi_{0} \in \mathrm{UC}\left(\mathbf{R}^{n}\right)$ such that $K_{0}=\left\{(x, y) \in \mathbf{R}^{n} \times \mathbf{R} ; \psi_{0}(x) \geq 0\right\}$. As usual level set method we have the uniqueness of $\{\psi \geq 0\}\left(=\left\{(t, x, y) \in[0, T) \times \mathbf{R}^{n} \times \mathbf{R} ; \psi(t, x, y) \geq 0\right\}\right)$ that implies the uniqueness of $L$-solution for a given $u_{0} \in \mathrm{USC}\left(\mathbf{R}^{n}\right)$.

Theorem 3.4. Assume that $H$ satisfies (H1)-(H4). For a given $u_{0} \in U S C\left(\mathbf{R}^{n}\right)$, there is a unique $L$-solution of (3.1a)-(3.1b).

If $u_{0}$ is bounded and periodic in $x$ and $\psi_{0}$ is restricted as functions periodic in $x$, then the uniqueness of $\{\psi \geq 0\}$ follows from the invariance lemma (Lemma 4.1) and the comparison principle provided that $\psi_{0}$ is constant for large $|y|$. For general case we further need monotone convergence result as in [IS].

Sketch of the proof. Assume that $\left\{\psi_{0}<0\right\}=\left\{\tilde{\psi}_{0}<0\right\}$ for $\psi_{0}, \tilde{\psi}_{0} \in \mathrm{UC}\left(\mathbf{R}^{n}\right)$. We would like to prove that $\{\psi<0\}=\{\tilde{\psi}<0\}$ if $\psi$ and $\tilde{\psi}$ is the solution of (3.3a)-(3.3b) with initial data $\psi_{0}$ and $\tilde{\psi}_{0}$, respectively. By the invariance (Lemma 4.1) we may assume that $\psi \leq 0, \tilde{\psi} \leq 0$ by taking minus part of $\psi$ and $\tilde{\psi}$. Let $\psi_{m}$ be the solution (3.3a)-(3.3b) with initial data $\psi_{0 m}=\max \left(\psi_{0}, m \tilde{\psi}_{0}\right)$ for $m=1,2, \ldots$ Since $m \tilde{\psi}$ also solves (3.3a), we see, by Theorem 2.1, that $\left\{\psi_{m}<0\right\} \subset\{\tilde{\psi}<0\}$. Since $\psi_{0 m} \geq \psi_{0}$, by Theorem 2.1 we have $\psi_{m} \geq \psi$. Moreover $\psi_{m 0} \downarrow \psi_{0}$ as $m \rightarrow \infty$. By $\psi_{m 0} \downarrow \psi_{0}$ we mean the monotone convergence, i.e., $\psi_{m 0}(x) \geq \psi_{m+10}(x)$ and $\lim _{m \rightarrow \infty} \psi_{m 0}(x)=\psi_{0}(x)$ for all $x \in \mathbf{R}^{N}$. The next monotone convergence lemma implies $\psi_{m} \downarrow \psi$. Since $\left\{\psi_{m}<0\right\} \subset\{\tilde{\psi}<0\}$, this implies that $\{\psi<0\} \subset\{\tilde{\psi}<0\}$. A symmetric argument implies the reverse inclusion.

Monotone Convergence Lemma. Assume that $F$ satisfies (F1)-(F4). Let $\psi_{m}$ be the solution of (2.3) with initial data $\psi_{m 0} \in U C\left(\mathbf{R}^{N}\right)$. Assume that $\psi_{m 0} \downarrow \psi_{0} \in$ $U C\left(\mathbf{R}^{N}\right)$ as $m \rightarrow \infty$. Then $\psi_{m} \downarrow \psi$ as $m \rightarrow \infty$ and $\psi$ is the solution of (2.3) with initial data $\psi_{0}$.

Sketch of the proof of the lemma. It is not difficult to prove that inf ${ }_{m \geq 1} \psi_{m}=\varphi$ is a subsolution. By Theorem 2.1 it is clear that $\psi_{m} \geq \psi$ so that $\varphi \geq \psi$, where $\psi$ is the 
solution of (2.3) with $\left.\psi\right|_{t=0}=\psi_{0}$. It suffices to prove $\psi_{1}=\varphi, \psi_{2}=\psi$ satisfies (2.4) to get $\varphi \leq \psi$ by Theorem 2.1 since $\psi_{1}$ and $\psi_{2}$ fulfill the growth condition (Theorem 2.2.). We use a barrier argument suggested by [IS].

To show (2.4) it suffices to find a family of supersolutions $\left\{w_{\varepsilon}^{y}(t, x) ; 0<\varepsilon<\right.$ $\left.1, y \in \mathbf{R}^{N}\right\}$ such that

$$
\begin{aligned}
& \varphi(t, x) \leq w_{\varepsilon}^{y}(t, x)+\alpha(\varepsilon), \quad t \in[0, T), x, y \in \mathbf{R}^{N}, \\
& \lim _{\delta \rightarrow 0} \sup \left\{w_{\varepsilon}^{y}(t, x) ;|x-y| \leq \delta, 0 \leq t \leq \delta\right\} \leq \psi_{0}(y)+\beta(\varepsilon), y \in \mathbf{R}^{N}
\end{aligned}
$$

for some $\alpha$ and $\beta$ depends only on $\varepsilon$ and $\alpha(\varepsilon) \rightarrow 0, \beta(\varepsilon) \rightarrow 0$ as $\varepsilon \rightarrow 0$. Indeed, if such $w_{\varepsilon}^{y}$ exists then

$$
\lim _{\delta \rightarrow 0} \sup _{\substack{|x-y| \leq \delta \\ 0 \leq t \leq \delta}}\left\{\varphi(t, x)-\psi_{0}(y)\right\} \leq \lim _{\delta \rightarrow 0} \sup _{\substack{|x-y| \leq \delta \\ 0 \leq t \leq \delta}}\left\{w_{\varepsilon}^{y}(t, x)+\alpha(\varepsilon)-u_{0}(y)\right\} \leq \alpha(\varepsilon)+\beta(\varepsilon)
$$

Since $\psi$ is uniformly continuous on $\left[0, T^{\prime}\right] \times \mathbf{R}^{N}, T^{\prime} \in(0, T)$ and since $0<\varepsilon<1$ is arbitrary, this yields (2.4).

It remains to construct $w_{\varepsilon}^{y}$. Such $w_{\varepsilon}^{y}$ is constructed by Ishii to show the existence (Theorem 2.2) by Perron's method developed in [I2]. For reader's convenience we reproduce its construction since it has not been written in English. Instead of considering (2.3) we may consider

$$
v_{t}+v+F\left(t, z, v, v_{z}\right)=0
$$

by changing $v=e^{-t} \psi$ with different $F$ but satisfying (F1)-(F4). For large $A, B>0$ it is easy to see that

$$
g^{+}(x)=A\left(|x|^{2}+1\right)^{1 / 2}+B
$$

is a supersolution of (*). We may assume that $g^{+} \geq \psi_{m 0}$ in $\mathbf{R}^{N}$ for all $m$ by taking $A, B$ larger since $\psi_{m 0} \downarrow \psi_{0}$ and $\sup _{x \in \mathrm{R}^{N}}\left|\psi_{m 0}(x)\right| /(|x|+1)<\infty$. Let $m_{0}$ be the modulus such that

$$
\psi_{0}(x)-\psi_{0}(y) \leq m_{0}(|x-y|), \quad x, y \in \mathbf{R}^{N}
$$


We set

$$
\begin{aligned}
& v_{\varepsilon}^{y}(t, x)=\psi_{0}(y)+m_{0}(\varepsilon)+B_{\varepsilon}|x-y|+C_{\varepsilon} t \\
& A_{\varepsilon}:=\sup \left\{g^{+}(x) ;|x| \leq 2+1 / \varepsilon\right\}, B_{\varepsilon}:=\max \left\{m_{0}(\varepsilon) / \varepsilon, 2 A_{\varepsilon}\right\} \\
& C_{\varepsilon}:=A_{\varepsilon}+\sup \left\{|H(t, x, r, \xi)| ; 0 \leq t \leq T,|x| \leq 2+1 / \varepsilon,|r| \leq A_{\varepsilon},|p| \leq B_{\varepsilon}\right\}
\end{aligned}
$$

It is not difficult to see that if $|y| \leq 1 / \varepsilon$, then $v_{\varepsilon}^{y}$ is a supersolution of (*) in $(0, T) \times B_{2+1 / \varepsilon}(0)$, where $B_{r}(z)$ is a closed ball centered at $y \in \mathbf{R}^{N}$. Moreover $v_{\varepsilon}^{y} \geq g^{+}$on $[0, T] \times\left(\mathbf{R}^{N} \backslash B_{1}(y)\right)$. Furthermore,

$$
v_{\varepsilon}^{x}(0, x)=\psi_{0}(x)+m_{0}(\varepsilon), v_{\varepsilon}^{y}(0, x) \geq \psi_{0}(x) \quad \text { for } x \in \mathbf{R}^{N}
$$

By the construction if we set

$$
w_{\varepsilon}^{y}(t, x)=\min \left\{v_{\varepsilon}^{y}(t, x), g^{+}(x)\right\}
$$

then $w_{\varepsilon}^{y}$ is a subsolution of $(*)$ in $(0, T) \times \mathbf{R}^{N}$. The property

$$
\lim _{\delta \rightarrow 0} \sup \left\{w_{\varepsilon}^{y}(t, x) ;|x-y| \leq \delta, 0 \leq t \leq \delta\right\} \leq \psi_{0}(y)+m_{0}(\varepsilon)
$$

immediately follows from the choice of $v_{\varepsilon}^{y}$. Since $\psi_{0}$ is continuous, the convergence $\psi_{m 0} \downarrow \psi_{0}$ is uniform on $B_{R}(0)$ for $R>0$ by Dini's theorem. Since $v_{\varepsilon}^{y}(0, x) \geq \psi_{0}(x)$, $x \in \mathbf{R}^{N}$ and since $v_{\varepsilon}^{y} \geq g^{+}$on $[0, T] \times\left(\mathbf{R}^{N} \backslash B_{1}(y)\right)$, we now observe that for any $\varepsilon \in(0,1)$ there is $k_{0}=k_{0}(\varepsilon)>0$ such that $w_{\varepsilon}^{y}+\varepsilon$ is a supersolution of $(*)$. Since $w_{\varepsilon}^{y}$ and $\psi_{m}$ are uniformly continuous on $\left[0, T^{\prime}\right] \times \mathbf{R}^{N}, T^{\prime} \in(0, T)$, the comparison principle (Theorem 2.1) implies

$$
\psi_{m} \leq w_{\varepsilon}^{y}+\varepsilon \quad \text { in }[0, T) \times \mathbf{R}^{N}
$$

for $y \in \mathbf{R}^{N}, m \geq k_{0}$ (The growth condition in Theorem 2.1 is trivially fulfilled.) Thus we have $\varphi \leq w_{\varepsilon}^{y}+\varepsilon$.

Remark 3.5. For the level set equation (3.3a) any constant is a solution so for solution we see $\sup _{x}|\psi(t, x)| \leq \sup _{x}\left|\psi_{0}(x)\right|$ for all $t \in[0, T)$. Since $\psi_{0}$ is taken in 
BUC for a set $K_{0}=\left\{\psi_{0} \geq 0\right\}$, one may restrict a class of $\psi$ in Definition 3.3 by restricting $\mathrm{UC}$ by BUC without restricting class of $L$-solutions.

Theorem 3.6 (Comparison). Assume that $H$ satisfies (H1)-(H4). Let $u$ and $v$ be the $L$-solution of (3.1a)-(3.1b) with initial data $u_{0}$ and $v_{0}$, respectively, where $u_{0}, v_{0} \in U S C\left(\mathbf{R}^{n}\right)$. If $u_{0} \leq v_{0}$ on $\mathbf{R}^{n}$, then $u \leq v$ on $(0, T) \times \mathbf{R}^{n}$.

This follows from the comparison principle (Theorem 2.1) for (3.3a) by choosing initial data $\psi_{0}$ as in $\S 3.1$.

\subsection{Consistency with other notions of solutions.}

Fortunately, our $L$-solution is consistent with classical notion of discontinuous viscosity solutions.

Theorem 3.7. Assume that $H$ satisfies (H1)-(H4).

(i) Let $\bar{u}$ be an $L$-solution of (3.1a) in $I \times \mathbf{R}^{n}$. Then $\bar{u}$ is a viscosity solution in $I \times \mathbf{R}^{n}$ of (3.1a) provided that $\bar{u}$ does not take $\pm \infty$.

(ii) Let $\bar{u}$ be the $L$-solution of (3.1a)-(3.1b) with initial data $u_{0} \in U C\left(\mathbf{R}^{n}\right)$. Then $\bar{u}$ is the unique viscosity solution which is uniformly continuous on $\left[0, T^{\prime}\right) \times \mathbf{R}^{n}$ for each $T^{\prime} \in(0, T)$ and $\left.\bar{u}\right|_{t=0}=u_{0}$.

Note that assumption (H1)-(H4) implies (F1)-(F4) with $F=H, n=N, x=z, p=\xi$, so for given $u_{0} \in \mathrm{UC}\left(\mathbf{R}^{N}\right)$ there is a unique viscosity solution for (3.1a)-(3.1b).

If we consider the initial value problem for $H$ which is concave in $p, \bar{u}$ is a bilateral viscosity solution.

Theorem 3.8. Assume that $H$ satisfies (H1)-(H4). Assume that $p \mapsto H(t, x, y, p)$ is concave. Let $\bar{u}$ be the $L$-solution of (3.1a)-(3.1b) with $u_{0} \in U S C\left(\mathbf{R}^{n}\right)$ and $u_{0}$ is bounded from above. Then $\bar{u}$ is a bilateral viscosity solution with initial data $u_{0}$. 
Since it is known that bilateral viscosity solution of (3.1a)-(3.1b) is unique, in this case for given initial data $u_{0}$ the unique bilateral viscosity solution is the $L$-solution. Thus, the existence of $L$-solution yields the unique bilateral viscosity solution.

We shall prove these properties in the next section.

Remark 3.9. Our $L$-solution $\bar{u}$ may take $+\infty$. However, if the initial data $u_{0}$ is bounded from above, $\bar{u}$ is bounded on $(0, T) \times \mathbf{R}^{n}$ by comparison (Theorem 3.6) with a spatially constant solution.

Remark 3.10 (Localization). It is possible to define an $L$-solution of (3.1a)-(3.1b) in $I \times \Omega$ by replacing $\mathbf{R}^{n}$ by an open set $\Omega$ in $\mathbf{R}^{n}$. However, it is not clear whether or not $\bar{u}$ is an $L$-solution in $I \times \mathbf{R}^{n}$ if $\bar{u}$ is an $L$-solution in $I \times \Omega$ for an open neighborhood $\Omega$ of arbitrary given point $x \in \mathbf{R}^{n}$. We do not study this problem in the present paper.

Remark 3.11 For a general equation (2.7) one can extend Lemma 3.2, Theorems $3.4,3.6,3.7$ if the level set equation (2.8) enjoys monotonicity in $y$, comparison, stability, monotone convergence and invariance properties (see Lemma 4.1 below). Such properties are valid for example for (2.9). There are many other examples for the second order level set equations that satisfies these properties as studied in [CGG1], [GGIS], [IS]; for more examples see [G] and references cited there.

There is no notion corresponding to bilateral viscosity solutions for the second order equations.

\section{Consistency}

We shall prove Theorems 3.7-3.8. We first prove a version of invariance lemma which is a key for the level set method ([CGG1], [ESp], [G]).

Lemma 4.1 (Invariance). Assume that $F$ satisfies (F1). Assume that $\psi$ is a subsolution (resp. supersolution) of (3.3a). Assume that $\theta$ is upper (resp. lower) 
semicontinuous and nondecreasing. Assume that $\theta \not \equiv-\infty$ (resp. $\theta \neq \equiv+\infty)$. Then $\theta \circ \psi$ is also a subsolution (resp. supersolution) of (3.3a), where $(\theta \circ \psi)(t, x)=\theta(\psi(t, x))$.

Proof. This is standard if $\theta$ is continuous ([CGG1], [ESp], [G]); see also [ESou] for the first order equations. Even if $\theta$ is merely upper semicontinuous so that right continuous, there is a sequence $\left\{\theta_{m}\right\}$ of continuous functions on $\mathbf{R}$ such that $\theta_{m}(\sigma) \downarrow$ $\theta(\sigma)$ (monotone convergence) for all $\sigma \in \mathbf{R}$. To find such a sequence for $\theta$ satisfying $\theta(\sigma)>-\infty$ for all $\sigma \in \mathbf{R}$, it is enough to take $\theta_{m}$ as a piecewise linear function so that its jump of derivatives is contained in $\left\{k / 2^{m} ; k\right.$ : integer $\}$ and $\theta_{m}\left(k / 2^{m}\right)=$ $\theta\left((k+1) / 2^{m}\right)$. If there is $\sigma_{0}$ such that $\theta\left(\sigma_{0}\right)>-\infty$ and $\theta(\sigma)=-\infty$ for $\sigma<\sigma_{0}$ then we may assume $\sigma_{0}=0$ by translation. We take $\theta_{m}(\sigma)=m \sigma+\theta\left(1 / 2^{m}\right)$ for $\sigma<0$ and $\theta_{m}\left(k / 2^{m}\right)=\theta\left((k+1) / 2^{m}\right)$ for $k=0,1,2$, such that its jump of derivatives is contained in $\left\{k / 2^{m} ; k\right.$ : positive integer $\}$. If $\psi$ is a subsolution, so is $\theta_{m} \circ \psi$. Since $\theta_{m} \circ \psi \downarrow \theta \circ \psi$, by stability of subsolution (eg. [CGG1]), we see $\theta \circ \psi$ is also a subsolution. The proof for supersolutions is symmetric.

Since $\theta$ is allowed to be discontinuous, we may take $\theta$ as

$$
I^{-}(\sigma)=\left\{\begin{array}{ll}
0 & \sigma \geq 0 \\
-\infty & \sigma<0,
\end{array} \quad I^{+}(\sigma)= \begin{cases}0 & \sigma \leq 0 \\
+\infty & \sigma>0 .\end{cases}\right.
$$

As a corollary of Lemma 4.1 we have:

Proposition 4.2. Assume that $F$ satisfies (F1). Assume that $\psi$ is a subsolution (resp. supersolution) of (3.3a). Then $I^{-} \circ \psi$ (resp. $I^{+} \circ \psi$ ) is also a subsolution (resp. supersolution) of (3.3a).

For a given continuous function $\Psi: Z \times \mathbf{R} \rightarrow \mathbf{R}$ we define functions $\underline{f}$ and $\bar{f}$ from $Z$ to $\mathbf{R} \cup\{ \pm \infty\}$ by

$$
\begin{aligned}
& \underline{f}(z)=\inf \{y \in \mathbf{R} ;(z, y) \in \overline{\{\Psi<0\}}\}, z \in Z, \\
& \bar{f}(z)=\sup \{y \in \mathbf{R} ; \Psi(z, y) \geq 0\}, z \in Z,
\end{aligned}
$$

where $\{\Psi<0\}=\{(z, y) \in Z \times \mathbf{R} ; \Psi(z, y)<0\}$ and $Z$ is a metric space. 
Lemma 4.3. Assume that $y \mapsto \Psi(z, y)$ is nonincreasing for all $z \in Z$. Then $(\bar{f})_{*}=\underline{f}$ on $Z$.

Proof. By definition it is clear that $\underline{f} \leq \bar{f}$ on $Z$. Since $\underline{f}$ is lower semicontinuous, we see $\underline{f} \leq(\bar{f})_{*}$ on $Z$.

We observe that $\Psi \geq 0$ in some neighborhood of $\left(z_{0}, y_{0}\right)$ if $y_{0}<(\bar{f})_{*}\left(z_{0}\right), z_{0} \in Z$. Assume that there would exist a sequence $\left(z_{j}, y_{j}\right) \rightarrow\left(z_{0}, y_{0}\right)$ in $Z \times \mathbf{R}$ and $\Psi\left(z_{j}, y_{j}\right)<$ 0 . Since $(\bar{f})_{*}$ is lower semicontinuous and $y_{0}<(\bar{f})_{*}\left(z_{0}\right)$, we see that $y_{j} \leq(\bar{f})_{*}\left(z_{j}\right)$ for sufficiently large $j$. From the monotonicity of $\Psi(z, y)$ in $y$ it now follows that $\Psi\left(z_{j}, \bar{f}\left(z_{j}\right)\right)<0$ for large $j$. This would contradict the definition of $\bar{f}$ so $\Psi \geq 0$ in some neighborhood of $\left(z_{0}, y_{0}\right)$ in $Z \times \mathbf{R}$ for all $y_{0}<(\bar{f})_{*}\left(z_{0}\right), z_{0} \in Z$.

Thus if $\underline{f}\left(z_{0}\right)<(\bar{f})_{*}\left(z_{0}\right)$ for some $z_{0} \in Z$, then $\Psi \geq 0$ in some neighborhood of $\left(z_{0}, \underline{f}\left(z_{0}\right)\right)$ which would contradict the definition of $\underline{f}$. We have thus proved $\underline{f}=(\bar{f})_{*}$. on $Z$.

Proof of Theorem 3.7. (i) We shall first show that $\bar{u}$ defined by (3.4) is a viscosity subsolution of (3.1a) in $I \times \mathbf{R}^{n}$. This is a variant of the slicing lemma in [GG1, Lemma 1], [GG2]. We reproduce the proof for completeness. By Proposition 4.2 $I^{-} \circ \psi$ is a subsolution of (3.3a) in $I \times \mathbf{R}^{n} \times \mathbf{R}$. Assume that

$$
\max _{I \times \mathbf{R}^{n}}(\bar{u}-\varphi)=(\bar{u}-\varphi)(\hat{t}, \hat{x})=0
$$

and $\varphi \in C^{1}\left(I \times \mathbf{R}^{n}\right)$. We set $\tilde{\varphi}(t, x, y)=\varphi(t, x)-y$, and observe that $I^{-} \circ \psi-\tilde{\varphi}$ attains its maximum 0 over $I \times \mathbf{R}^{n} \times \mathbf{R}$ at $(\hat{t}, \hat{x}, \hat{y})$ with $\hat{y}=\bar{u}(\hat{t}, \hat{x})$. Since $I^{-} \circ \psi$ is a subsolution of (3.3a) in $I \times \mathbf{R}^{n} \times \mathbf{R}$, we have

$$
\varphi_{t}(\hat{t}, \hat{x})+1 \cdot H\left(\hat{x}, \hat{y}, \varphi_{x}(\hat{t}, \hat{x})\right) \leq 0
$$

We have thus proved that $\bar{u}$ is a subsolution of (3.1a) in $I \times \mathbf{R}^{n}$.

We next prove that $\bar{u}$ is a supersolution of (3.1a). The proof is more complicated than that for subsolution. We set

$$
\underline{u}(t, x)=\inf \{y ;(t, x, y) \in \overline{\{\psi<0\}}\}, \quad t \in I, x \in \mathbf{R}^{n}
$$


By Lemma 4.3 we see $(\bar{u})_{*}=\underline{u}$. Thus it suffices to prove that $\underline{u}$ is a supersolution of (3.1a). Since $\psi$ is a supersolution of (3.3a) so is $I^{+} \circ(\psi+1 / m)$ by Proposition 4.2 for $m=1,2, \cdots$. Since the infimum of a family of supersolutions is a supersolution [CIL], [CGG1], $W(t, x, y)=\inf \left\{\left(I^{+} \circ(\psi+1 / m)\right)(t, x, y), m=1,2, \cdots\right\}$ is a supersolution of (3.3a). By definition

$$
W(t, x, y)= \begin{cases}+\infty & \text { if } \quad(t, x, y) \in\{\psi \geq 0\} \\ 0 & \text { if } \quad(t, x, y) \in\{\psi<0\}\end{cases}
$$

and

$$
W_{*}(t, x, y)= \begin{cases}+\infty & \text { if } \quad(t, x, y) \in \operatorname{int}\{\psi \geq 0\} \\ 0 & \text { if } \quad(t, x, y) \in \overline{\{\psi<0\}} .\end{cases}
$$

Since $W_{*}$ is a supersolution of (3.3a), as in the proof of showing that $\bar{u}$ is a subsolution we observe that $\underline{u}$ is a supersolution of (3.1a).

We have thus proved that $\bar{u}$ is a sub and supersolutions of (3.1a) in $I \times \mathbf{R}^{n}$, so $\bar{u}$ is a viscosity solution of (3.1a) in $I \times \mathbf{R}^{n}$.

(ii) If $u_{0}$ is uniformly continuous, there is a unique viscosity solution $u$ of (3.1a)(3.1b) which is uniformly continuous on $\left[0, T^{\prime}\right] \times \mathbf{R}^{n}$ for any $T^{\prime} \in(0, T)$. If we set $\psi(t, x, y)=u(t, x)-y$, then $\psi$ solves (3.3a)-(3.3b) with $\left\{\psi_{0} \geq 0\right\}=K_{0}$. Since $\psi$ is uniformly continuous on each $\left[0, T^{\prime}\right] \times \mathbf{R}^{n}$, we see that $u$ is the unique $L$-solution of (3.1a), (3.1b).

Proof of Theorem 3.8. We recall the property that the bilateral viscosity solution is given as a monotone limit of continuous viscosity solutions as proved in [BJ1].

Let $\left\{u_{0 \varepsilon}\right\}$ be a sequence of uniformly continuous functions on $\mathbf{R}^{n}$ with $u_{0 \varepsilon} \downarrow u_{0}$ as $\varepsilon \rightarrow 0$. Let $u_{\varepsilon}$ be the unique continuous viscosity solution of (3.1a)-(3.1b) (which is uniformly continuous on each $\left[0, T^{\prime}\right) \times \mathbf{R}^{n}$ with $\left.T^{\prime} \in(0, T)\right)$. If $u_{\varepsilon} \downarrow u$, then $u$ is the unique bilateral viscosity solution of (3.1a)-(3.1b).

For $u_{0} \in \mathrm{USC}\left(\mathbf{R}^{n}\right)$ we take sup-convolution

$$
u_{0}^{\varepsilon}(x)=\sup _{y \in \mathbf{R}^{n}}\left\{u_{0}(y)-|x-y|^{2} / \varepsilon^{2}\right\}, \quad x \in \mathbf{R}^{n}
$$

and define $u_{0 \varepsilon}=u_{0}^{\varepsilon}+\varepsilon$. Note that $u_{0 \varepsilon_{1}} \geq u_{0 \varepsilon_{2}}+\left(\varepsilon_{1}-\varepsilon_{2}\right)$ if $\varepsilon_{1}>\varepsilon_{2}$. Since $u_{0}^{\varepsilon}$ is Lipschitz continuous on $\mathbf{R}^{n}$, the next lemma completes the proof. 
Lemma 4.4. Assume that $u_{0 \varepsilon} \downarrow u_{0} \in U S C\left(\mathbf{R}^{n}\right)$ as $\varepsilon \rightarrow 0$ and that $u_{0 \varepsilon}$ is uniformly continuous on $\mathbf{R}^{n}$. Assume that $u_{0 \varepsilon} \geq u_{0 \varepsilon^{\prime}}+\varepsilon-\varepsilon^{\prime}$ for $\varepsilon>\varepsilon^{\prime}>0$. Let $u_{\varepsilon}$ be the solution of (3.1a)-(3.1b) with $u_{0}=u_{0 \varepsilon}$. Then $\lim _{\varepsilon \rightarrow 0} u_{\varepsilon}$ is an L-solution of (3.1a)-(3.1b) (so that it agrees with $\bar{u}$ ).

Proof. We set

$$
\psi_{0}(x, y)= \begin{cases}-\left\{\inf \left\{\varepsilon ; y \leq u_{0 \varepsilon}(x)\right\} \wedge 1\right\}, & (x, y) \notin K_{0} \\ 0, & (x, y) \in K_{0}\end{cases}
$$

where $K_{0}=\left\{(x, y) \in \mathbf{R}^{n} \times \mathbf{R} ; y \leq u_{0}(x)\right\}$.

It turns out that $\psi_{0} \in \mathrm{BUC}\left(\mathbf{R}^{n}\right)$ which we shall prove at the end of the proof. Let $\psi$ be the unique viscosity continuous solution of (3.3a)-(3.3b) with initial data $\psi_{0}$. Since

$$
\left\{\psi_{0} \geq-\varepsilon\right\}=\left\{(x, y) ; y \leq u_{0 \varepsilon}(x)\right\}
$$

for $\varepsilon>0$, the $L$-solution of (3.1a)-(3.1b) with $\left.u\right|_{t=0}=u_{0 \varepsilon}$ given by

$$
u_{\varepsilon}(t, x)=\sup \{y ; \psi(t, x, y) \geq-\varepsilon\}, \quad t>0, x \in \mathbf{R}^{n}
$$

is the unique continuous viscosity solution of (3.1a)-(3.1b) with initial data $u_{0 \varepsilon}$ by Theorem 3.7 (ii). The $L$-solution of (3.1a)-(3.1b) with $\left.u\right|_{t=0}=u_{0}$ is given by

$$
u(t, x)=\sup \{y ; \psi(t, x, y) \geq 0\}
$$

By definition $u_{\varepsilon} \downarrow u$ as $\varepsilon \rightarrow 0$. Thus $u$ is the bilateral viscosity solution with initial data $u_{0}$.

It remains to prove that $\psi_{0} \in \mathrm{BUC}\left(\mathbf{R}^{n}\right)$. The boundedness of $\psi_{0}$ is clear by definition. Suppose that $\psi_{0}$ were not uniformly continuous in $\mathbf{R}^{n}$. Then there would exist sequences $\left\{\left(x_{j}, y_{j}\right)\right\}_{j=1}^{\infty},\left\{\left(\tilde{x}_{j}, \tilde{y}_{j}\right)\right\}_{j=1}^{\infty} \subset \mathbf{R}^{n} \times \mathbf{R}$ and $\eta>0$ that satisfies

$$
\begin{aligned}
& \left|\psi\left(x_{j}, y_{j}\right)-\psi\left(\tilde{x}_{j}, \tilde{y}_{j}\right)\right| \geq \eta \quad \text { for all } j \geq 1 ; \\
& \lim _{j \rightarrow \infty}\left|x_{j}-\tilde{x}_{j}\right|=0, \lim _{j \rightarrow \infty}\left|y_{j}-\tilde{y}_{j}\right|=0 .
\end{aligned}
$$


We may assume that $-1 \leq \psi\left(x_{j}, y_{j}\right)<\psi\left(\tilde{x}_{j}, \tilde{y}_{j}\right) \leq 0$. By the definition of $\psi\left(x_{j}, y_{j}\right)=$ $-\varepsilon_{j}$ we observe that

$$
\begin{array}{ll}
y_{j} \leq f_{\varepsilon}\left(x_{j}\right) & \text { for } \varepsilon \in\left(\varepsilon_{j}, 1\right] \text { and } \\
y_{j}>f_{\varepsilon}\left(x_{j}\right) & \text { for } \varepsilon \in\left(0, \varepsilon_{j}\right),
\end{array}
$$

where $f=u_{0}$ and $f_{\varepsilon}=f^{\varepsilon}+\varepsilon$. We may assume that $\left\{\varepsilon_{j}\right\}_{j=1}^{\infty}$ and $\left\{\tilde{\varepsilon}_{j}\right\}_{j=1}^{\infty}$ for $\tilde{\varepsilon}_{j}=-\psi\left(\tilde{x}_{j}, \tilde{y}_{j}\right)$ converges to $a$ and $b \in[0,1]$ by taking a subsequence if necessary. Since $a \geq b+\eta$, we may assume that there would exist a constant $\delta>0$ (independent of $j$ ) that satisfies $\varepsilon_{j}-\delta>b+\delta$. By the definition of $\varepsilon_{j}$ it follows from monotonicity of $u_{0}^{\varepsilon}$ in $\varepsilon$ that

$$
y_{j}>f_{\varepsilon_{j}-\delta}\left(x_{j}\right)>f_{b+\delta}\left(x_{j}\right) \geq f_{b}\left(x_{j}\right)+\delta
$$

By the definition of $\tilde{\varepsilon}_{j}$ we also see that for large $j$

$$
\tilde{y}_{j} \leq f_{b+\delta}\left(\tilde{x}_{j}\right)
$$

Combining these inequalities we observe that

$$
\begin{aligned}
y_{j}-\tilde{y}_{j} & \geq f_{b+\delta}\left(x_{j}\right)-f_{b+\delta}\left(\tilde{x}_{j}\right)+\delta \\
& \geq-m_{b+\delta}\left(\left|x_{j}-\tilde{x}_{j}\right|\right)+\delta
\end{aligned}
$$

where $m_{\varepsilon}$ is a modulus of continuity of $f_{\varepsilon}$. Since $y_{j}-\tilde{y}_{j} \rightarrow 0$ and $x_{j}-\tilde{x}_{j} \rightarrow 0$ as $j \rightarrow \infty$, this yields a contradiction.

\section{Maximality of $L$-solutions}

Our $L$-solution can be characterized as a maximal subsolution whose initial data is less than the given initial data at least when the speed $u_{t}$ is controlled by the gradient $u_{x}$ of $u$. 
Theorem 5.1. Assume that $H$ satisfies (H1)-(H4). Assume that $\sup _{p, r, x} \mid H$ $(x, r, p) /(|p|+1) \mid<\infty$. For a given $u_{0} \in$ USC $\left(\mathbf{R}^{n}\right)$ let $u$ be the $L$-solution of (3.1a)-(3.1b). Then

$$
\begin{aligned}
u(t, x)= & \sup \left\{\left(v(t, x) ; v \text { is a subsolution of }(3.1 a) \text { in }(0, T) \times \mathbf{R}^{n}\right.\right. \\
& \text { with } \left.\left.v^{*}\right|_{t=0} \leq u_{0} \text { on } \mathbf{R}^{n}\right\}
\end{aligned}
$$

for $t \in[0, T), x \in \mathbf{R}^{n}$.

Proof. Since $u$ itself is a subsolution of (3.1a) in $(0, T) \times \mathbf{R}^{n}$ with $u^{*}=\left.u\right|_{t=0} \leq u_{0}$, it suffices to prove that $u$ is greater than or equal to the right hand side of (5.1).

We again use $u_{0 \varepsilon}=u_{0}^{\varepsilon}+\varepsilon$ where $u_{0}^{\varepsilon}$ is the sup-convolution of $u_{0}$. Let $u_{\varepsilon}$ be the solution of (3.1a), (3.2b) with initial data $u_{0 \varepsilon}$. If we prove that for $\varepsilon>0$

$$
\varliminf_{\substack{t \rightarrow 0 \\ s \rightarrow 0}} \operatorname{dist}\left(K(t), \operatorname{graph} u_{\varepsilon}(s, \cdot)\right)>0
$$

with

$$
K(t)=\{(x, y) ; y \leq u(t, x)\},
$$

then by comparison principle for the level set equation (Theorem 2.1), we see

$$
v(t, x) \leq u_{\varepsilon}(t, x)
$$

By Lemma 4.4 we see $u_{\varepsilon} \downarrow u$. Thus we obtain $v \leq u$.

It remains to prove (5.2). Since $|H(x, r, p) /(|p|+1)|$ is bounded, we see

$$
\lim _{t \rightarrow 0} \sup _{x \in K(t)} \operatorname{dist}(x, K(0))=0 .
$$

Since $u_{\varepsilon}$ is uniformly continuous in $\mathbf{R}^{n} \times\left[0, T^{\prime}\right)$ for each $T^{\prime} \in(0, T)$, we see

$$
\lim _{s \rightarrow 0} \sup \left\{\operatorname{dist}\left(y, \operatorname{graph} u_{0 \varepsilon}\right) ; y \in \operatorname{graph} u_{\varepsilon}(s, \cdot)\right\}=0 .
$$

By the definition of $u_{0 \varepsilon}$ it is not diffcult to see that

$$
\operatorname{dist}\left(K(0), \text { graph } u_{0 \varepsilon}\right)>0 .
$$


We have thus proved (5.2).

\section{Right accessibility}

We give a simple criterion for right accessibility.

\subsection{The Case $H=H(p)$ is homogeneous}

Lemma 6.1. Assume that $F \in C\left(\mathbf{R}^{N}\right)$ is positively homogeneous of degree one. Let $A$ be a closed convex set in $\mathbf{R}^{N}$. Let $w$ be the $L$-solution of

$$
\begin{gathered}
w_{t}+F\left(w_{z}\right)=0, \quad z \in \mathbf{R}^{N}, t>0 \\
\left.w\right|_{t=0}=w_{0}
\end{gathered}
$$

with $w_{0}(z)=0, z \in A$ and $\sup \left\{w_{0}(z) ; \operatorname{dist}(z, A) \geq \delta\right\}<0$ for $\delta>0$. Then

$$
w(t, z)= \begin{cases}0 & z \in A+t W_{\alpha} \\ <0 & \text { otherwise. }\end{cases}
$$

Here

$$
W_{\alpha}=\left\{z \in \mathbf{R}^{N} ; \sup _{|p|=1}(z \cdot p-\alpha(p)) \leq 0\right\}, \alpha(p)=-F(-p)
$$

For sets $A, B$ in $\mathbf{R}^{N}$ by $A+B$ we mean the set of all vector sum $a+b$ of $a \in A$ and $b \in B$ in $\mathbf{R}^{N}$. If one of $A$ and $B$ is empty, we interpret $A+B$ as an empty set. The set $W_{\alpha}$ is often called the Wulff shape with respect to $\alpha$ if $\alpha$ is positive. The set $W_{\alpha}$ is closed and convex but may be empty. For example if $F(p)=|p|$, then $W_{\alpha}=\emptyset$. On the other hand if $\alpha$ is convex, then $W_{\alpha}$ is not empty. Since $W_{\alpha}=W_{\hat{\alpha}}$ for the convex hull $\hat{\alpha}$ of $\alpha, W_{\alpha}=\emptyset$ if and only if $\hat{\alpha} \equiv-\infty$ (or equivalently $\hat{\alpha}$ takes $-\infty$ at some point.) (For example for $\alpha(p)=-|p|$, we see $\hat{\alpha} \equiv-\infty$ so that $W_{\alpha}=\emptyset$.) As an application of Lemma 6.1 we have:

Theorem 6.2. If $H \in C\left(\mathbf{R}^{n}\right)$ is positively homogeneous degree of one, and independent of $x$ and $u$, then an $L$-solution of (3.1a)-(3.1b) is right accessible for 
any $u_{0} \in U S C\left(\mathbf{R}^{n}\right)$ if and only if $W_{\alpha} \neq \emptyset$, where $\alpha(p)=-H(-p)$ and $N=n$. In particular, for concave $H$ the $L$-solution is always right accessible for any $u_{0} \in$ USC $\left(\mathbf{R}^{n}\right)$.

We shall prove Lemma 6.1 and Theorem 6.2 in the rest of this section.

Lemma 6.3. Let $F \in C\left(\mathbf{R}^{N}\right)$ be positively homogeneous of degree one. For $x_{0} \in W_{\alpha}$ with $\alpha(p)=-F(-p)$ let $w$ denote a function of $z \in \mathbf{R}^{N}, t \geq 0$ defined by

$$
w(t, z)= \begin{cases}0 & z=t x_{0} \\ -\infty & z \neq t x_{0}\end{cases}
$$

Then $w$ is subsolution of $(6.1 \mathrm{a})$ in $Q=(0, \infty) \times \mathbf{R}^{N}$.

Proof of Lemma 6.3. If $\varphi \in C^{1}(Q)$ and $(\hat{t}, \hat{z}) \in Q$ satisfies

$$
\max _{Q}(w-\varphi)=(w-\varphi)(\hat{t}, \hat{z})=0
$$

then by definition of $w$ we see that $\hat{z}=\hat{t} x_{0}$. Since $w\left(t, t x_{0}\right)=0$, the function $\rho(t)=\varphi\left(t, t x_{0}\right)$ attains its minimum at $t=\hat{t}$. We thus observe that

$$
0=\frac{d \rho}{d t}(\hat{t})=\hat{\varphi}_{t}+x_{0} \cdot \hat{\varphi}_{z}=\hat{\varphi}_{t}-\left|\hat{\varphi}_{z}\right|\left(x_{0} \cdot\left(-\hat{\varphi}_{z}\right) /\left|\hat{\varphi}_{z}\right|\right)
$$

where $\hat{\varphi}_{t}=\varphi_{t}(\hat{t}, \hat{z}), \hat{\varphi}_{z}=\varphi_{z}(\hat{t}, \hat{z})$. By definition of $W_{\alpha}$ the inequality $x_{0} \cdot p \leq \alpha(p)$ holds for all $p \in \mathbf{R}^{N}$ satisfying $|p|=1$. We thus conclude that

$$
0 \geq \hat{\varphi}_{t}-\left|\hat{\varphi}_{z}\right| \alpha\left(-\hat{\varphi}_{z} /\left|\hat{\varphi}_{z}\right|\right)=\hat{\varphi}_{t}+F\left(\hat{\varphi}_{z}\right)
$$

so that $w$ is a subsolution of $w_{t}+F\left(w_{z}\right)=0$.

Proof of Lemma 6.1. By Lemma 6.3 we see that

$$
w\left(t, z ; z_{0}\right)= \begin{cases}0 & z=t x_{0}+z_{0} \\ -\infty & z \neq t x_{0}+z_{0}\end{cases}
$$


for $z_{0} \in \mathbf{R}^{N}, x_{0} \in W_{\alpha}$ is a subsolution of (6.1a) in $Q$. By the maximality of an $L$-solution $w$ of $(6.1 \mathrm{a}),(6.1 \mathrm{~b})$ we have $w\left(t, z ; z_{0}\right) \leq w(t, z)$ for all $(t, z) \in Q$ provided that $z_{0} \in A$. This implies

$$
w(t, z) \geq 0 \text { for } z \in A+t W_{\alpha}
$$

and actually $w(t, z)=0$ for $z \in A+t W_{\alpha}$ since $w_{0} \leq 0$ implies $w \leq 0$ for $(t, z) \in Q$.

It remains to prove that $w(t, z)=0$ implies that $z \in A+t W_{\alpha}$. By assumption on $w$ there exists $\tilde{w}_{0} \in \mathrm{BUC}\left(\mathbf{R}^{N}\right)$ so that $w_{0} \leq \tilde{w}_{0} \leq 0$ and the zero set of $\tilde{w}_{0}$ agrees with that of $w_{0}$. Since the viscosity solution $\tilde{w}$ of $(6.1 \mathrm{a}),(6.1 \mathrm{~b})$ with initial data $\tilde{w}_{0}$ is always the $L$-solution (Theorem 3.7 ), we have, by comparison (Theorem 3.6) $w \leq \tilde{w}$. Thus it suffices to prove $\tilde{w}(t, z)=0$ implies that $z \in A+t W_{\alpha}$. In other words we may assume that $w_{0} \in \mathrm{BUC}\left(\mathbf{R}^{N}\right)$. By the uniqueness of the level set, the set $\{w=0\}$ is determined by $A$ and independent of the choice of $w_{0}$ (cf. the proof of Theorem 3.4.).

Suppose that $w_{0}, \bar{w}_{0} \in \mathrm{BUC}\left(\mathbf{R}^{N}\right)$ such that $w_{0} \leq 0, \bar{w}_{0} \leq 0$ and $A=\left\{w_{0}=\right.$ $0\} \subset A^{\prime}=\left\{\bar{w}_{0}=0\right\}$. Then by the uniqueness of the level set and the comparison principle (Theorem 2.1) we have

$$
\{w=0\} \subset\{\bar{w}=0\} \text { in } Q
$$

where $w$ and $\bar{w}$ is the viscosity solution of $(6.1 \mathrm{a}),(6.1 \mathrm{~b})$ with initial data $w_{0}$ and $\bar{w}_{0}$, respectively. Since $A$ is convex, there exists a family $\mathcal{S}$ of half spaces that satisfies

$$
A=\bigcap\{S ; S \in \mathcal{S}\}
$$

Thus to show that $(t, z) \in\{w=0\}$ implies $z \in A+t W_{\alpha}$ it suffices to prove the assertion of Lemma 6.1 for a half space with some $w_{0} \in \operatorname{BUC}\left(\mathbf{R}^{N}\right)$.

We may assume that

$$
A=\left\{z \in \mathbf{R}^{N} ; z \cdot p_{0} \leq 0\right\}
$$

with some $p_{0} \in \mathbf{R}^{N},\left|p_{0}\right|=1$. Since

$$
u(t, z)=-z \cdot p_{0}+\alpha\left(p_{0}\right) t
$$


solves (6.1a), by the invariance (Lemma 4.1) $w=\min (u, 0)$ also solves (6.1a). We shall prove that

$$
\left\{z \in \mathbf{R}^{N} ; z \cdot p_{0} \leq t \alpha\left(p_{0}\right)\right\}=A+t W_{\alpha}, \quad t \geq 0
$$

We may assume that $p_{0}=(1,0, \ldots, 0)$ by a rotation of coordinates. By definition for $t>0$

$$
\begin{aligned}
A+t W_{\alpha}=A+W_{\alpha t}= & \left\{\left(z_{1}+x_{1}, z^{\prime}+x^{\prime}\right) \in \mathbf{R} \times \mathbf{R}^{N-1} ; z_{1} \leq 0, z^{\prime} \in \mathbf{R}^{N-1}\right. \\
& \text { and } \left.\sup _{|\boldsymbol{p}|=1}(x \cdot p-t \alpha(p)) \leq 0 \text { with } x=\left(x_{1}, x^{\prime}\right)\right\}
\end{aligned}
$$

Note that

$$
\sup _{|p|=1}(x \cdot p-t \alpha(p)) \leq 0 \text { for some } x^{\prime} \in \mathbf{R}^{N-1}
$$

is equivalent to say

$$
\sup _{\left|p_{1}\right|=1}\left(x_{1} p_{1}-t \alpha\left(p_{1}, 0, \ldots, 0\right)\right) \leq 0 .
$$

Since $z^{\prime}$ is arbitrary, this implies

$$
\begin{aligned}
A+t W_{\alpha}= & \left\{\left(z_{1}+x_{1}, z^{\prime}\right) \in \mathbf{R} \times \mathbf{R}^{N-1} ; z_{1} \leq 0, z^{\prime} \in \mathbf{R}^{N-1}\right. \\
& \text { and } \left.\sup _{\left|p_{1}\right|=1}\left(x_{1} p_{1}-t \alpha\left(p_{1}, 0, \ldots, 0\right)\right) \leq 0\right\} .
\end{aligned}
$$

Since $z_{1}$ is an arbitrary negative number, we see

$$
\begin{aligned}
A+t W_{\alpha}= & \left\{\left(z_{1}+x_{1}, z^{\prime}\right) \in \mathbf{R} \times \mathbf{R}^{N-1} ; z_{1} \leq 0, z^{\prime} \in \mathbf{R}^{N-1}\right. \\
& \text { and } \left.x_{1} \leq t \alpha(1,0, \ldots, 0)\right\}
\end{aligned}
$$

which proves (6.2). By (6.2) we see that $(t, z) \in\{w=0\}$ is equivalent to $z \in A+t W_{\alpha}$. We have proved the assertion of Lemma 6.1 when $A$ is a half space and $w_{0} \in$ BUC $\left(\mathbf{R}^{N}\right)$. Thus the proof is now complete.

Proof of Theorem 6.2. We consider $u_{t}+H\left(u_{x}\right)=0$ with initial data

$$
u_{0}^{a, \sigma}(x)= \begin{cases}\sigma & x=a \\ -\infty & x \neq a .\end{cases}
$$


where $a \in \mathbf{R}^{N}, \sigma \in \mathbf{R} \cup\{+\infty\}$. Let $u^{a, \sigma}$ denote the $L$-solution of this initialvalue problem. Applying Lemma 6.1 for the level set equation (3.3a) with $A=$ $\{a\} \times(-\infty, \sigma]\left(\cap \mathbf{R}^{n+1}\right)$ we see that

$$
u^{a, \sigma}(t, x)= \begin{cases}\sigma & x \in a+t W_{\alpha} \\ -\infty & x \notin a+t W_{\alpha} .\end{cases}
$$

Since $W_{-F(-)}=\left\{(x, y) ; x \in W_{\alpha}, y=0\right\}$, where $F(p, q)=H(p)$. Evidently, $u^{a, \sigma}$ is right accessible at $t=0$ if and only if $W_{\alpha}=\emptyset$. Thus to prove Theorem 6.2 it suffices to prove the next lemma (Lemma 6.5) which holds without assuming the homogeneity of $H$.

Our solution formula (6.3) immediately yields:

Corollary 6.4. Let $u$ be the $L$-solution if (3.1a)-(3.1b) with $H(p)=|p|$ and $u_{0}=u_{0}^{a, \sigma}$ (defined in the proof of Theorem 6.2). Then $u(x, t)=-\infty$ for $x \in \mathbf{R}^{n}$ and $t>0$.

Lemma 6.5. Assume that $H$ satisfies (H1)-(H4). Let $u^{a, \sigma}$ be the $L$-solution of (3.1a)-(3.1b) with initial data $u_{0}^{a, \sigma}$. If $u_{0}^{a, \sigma}$ is right accessible at $t=0$ for all $a \in \mathbf{R}^{n}$ and $\sigma \in \mathbf{R} \cup\{+\infty\}$, then the $L$-solution $u$ of (3.1a)-(3.1b) with any initial data $u_{0} \in$ $U S C\left(\mathbf{R}^{n}\right)$ is also right accessible at $t=0$.

Proof. For $a \in \mathbf{R}^{n}$ and $\sigma=u_{0}(a)$ we see $u_{0}^{a, \sigma} \leq u_{0}$. By the comparison principle (Theorem 3.6) for $L$-solutions we have

$$
u^{a, \sigma}(t, x) \leq u(t, x) \quad \text { in }(0, \infty) \times \mathbf{R}^{n}
$$

Since $u^{a, \sigma}$ is right accessible, there is a sequence $\left(t_{k}, x_{k}\right) \rightarrow(0, a)(k \rightarrow \infty)$ such that

$$
u_{0}(a)=\lim _{k \rightarrow \infty} u^{a, \sigma}\left(t_{k}, x_{k}\right)
$$

Since $u^{a, \sigma} \leq u$ and $u$ is upper semicontinuous, this implies

$$
u_{0}(a) \leq \varliminf_{k \rightarrow \infty} u\left(t_{k}, x_{k}\right) \leq \varlimsup_{k \rightarrow \infty} u\left(t_{k}, x_{k}\right) \leq u_{0}(a)
$$


Thus $u$ is right accessible.

6.2 The case $H=H(p)$ is inhomogeneous

Lemma 6.1 is important to study $L$-solutions with initial data $u_{0}^{a, \sigma}$ when $H$ is not positively homogeneous of degree one.

Lemma 6.6. Assume that $H \in C\left(\mathbf{R}^{n}\right)$ satisfies (H1) and is independent of $x, t$ and $u$. Let $u$ be the $L$-solution of (3.1a), (3.1b) with $u_{0}(x)=0$ for $x=0 ; u_{0}(x)=-\infty$ for $x \neq 0$. Then

$$
u(t, x)=\sup \left\{y ;(x, y) \in A+t W_{\beta}\right\}, \quad t \geq 0, x \in \mathbf{R}^{n}
$$

with $A=\{0\} \times(-\infty, 0]$ and $\beta(p, q)=-F(-p,-q), p \in \mathbf{R}^{n}, q \in \mathbf{R}$ where $F$ is defined by (2.6).

This follows from Lemma 6.1 with $N=n+1$ for the level set equation (2.2). It is not difficult to see that $u$ is accessible if and only if $W_{\beta} \neq \emptyset$. By using Lemma 6.5 we easily extend Theorem 6.2 to inhomogeneous $H$.

Theorem 6.7. Assume that $H \in C\left(\mathbf{R}^{n}\right)$ satisfies $(H 1)$ and is independent of $x, t$ and $u$. Then an $L$-solution of (3.1a)-(3.1b) is right accessible for any $u_{0} \in U S C\left(\mathbf{R}^{n}\right)$ if and only if $W_{\beta} \neq \emptyset$. In particular, for concave $H$ the $L$-solution is always right accessible for any $u_{0} \in U S C\left(\mathbf{R}^{n}\right)$.

Remark 6.8. For the second order problem the zero set of an $L$-solution with $w_{0}$ in Lemma 6.1 is not explicitly written. Moreover, if $G$ in (2.7) is geometric in the sense of [CGG1] and strictly parabolic in the direction orthogonal to $\nabla u$, then the zero set of the $L$-solution $w$ always disappear when $A$ is a point or $\{a\} \times(-\infty, \sigma]$. Thus, the $L$-solution is not right accessible when initial data is $u_{0}^{a, \sigma}$ given in the proof of Theorem 6.2. A typical example is the level set mean curvature flow equation $w_{t}-|D w| \operatorname{Div}(D w /|D w|)=0$. We do not pursue the accessibility problem for the second order problems in the present paper. 
Remark 6.9. In [BJ1, Theorem 3.8] the accessibility of their bilateral solution is proved for fairly general Hamiltonian $H(t, x, p)$ which is concave in $p$. However, it seems that their general approximating argument needs more explanation. In the second paragraph of the proof of [BJ1, Theorem 3.8, p.1736] it is asserted that 'By diagonalization, at least on a subsequence, $u_{i}^{\varepsilon} \downarrow u^{\varepsilon}$ and $g_{i}^{\varepsilon} \downarrow g^{\varepsilon}$, as $i \rightarrow \infty$ uniformly in $\varepsilon, \ldots$ ' However, the uniformity in $\varepsilon$ is questionable. If such argument would work, then we would get the accessibility always for our $L$-solution since $L$-solution is also given as approximation from above as in Lemma 4.4. This is of course absurd. More specific feature of the equation should be utilized and an alternative proof reflecting underlying control problems is essentially given in [BC, p.351]; see also [BJ2]. Our Theorem 6.7 also gives a direct alternate proof when $H$ is independent of $t, x$ and $u$.

\section{ACKNOWLEDGEMENT}

The first author is partly supported by the Grant-in-Aid for Scientific Research (No. 10304010) from the Japan Society for the Promotion of Science. The authors are grateful to Professor Martino Bardi for his interest and informing them of his recent results. The authors are also grateful to Professor Thai Son Nguyen Duy for letting them know that (1.3a) was used in [S].

\section{REFERENCES}

[BC] M. Bardi and I. Capuzzo-Dolcetta, Optimal Control and Viscosity Solutions of Hamiltonian-Jacobi-Bellman Equation, Systems \& Control: Foundations \& Applications, Birkhäuser, Boston, (1997).

[BCESS] M. Bardi, M. G. Crandall, L. C. Evans, H. M. Soner, P. E. Souganidis, Viscosity Solutions and Applications, Springer Lecture Notes in Math., 1660(1997). 
[B1] G. Barles, Discontinuous viscosity solutions of first-order Hamilton-Jacobi equations, Nonlinear Anal., TMA 20(1993), 1123-1134.

[B2] G. Barles, Solutions de Viscosité des Equations de Hamilton-Jacobi, Mathématiques \& Applications, vol.17, Springer-Verlag, Paris, (1994).

[BSS] G. Barles, H. M. Soner and P. E. Souganidis, Front propagation and phase field. theory, SIAM J. Cont. Opt. 31(1993), 439-469.

[BJ1] E. N. Barron and R. Jensen, Semicontinuous viscosity solutions of HamiltonJacobi equations with convex Hamiltonians, Commun. in Partial Differential Equations, 15(1990), 1713-1742.

[BJ2] E. N. Barron and R. Jensen, Optimal control and semicontinuous viscosity solutions, Proc. AMS, 113(1991), 397-402.

[CGG1] Y.-G. Chen, Y. Giga and S. Goto, Uniqueness and existence of viscosity solutions of generalized mean curvature flow equation, J. Differential Geometry, 33(1991), 749-786. (Announcement: Proc. Japan Acad., Ser. A., 65(1989), 207-210.)

[CGG2] Y.-G. Chen, Y. Giga and S. Goto, Remarks on viscosity solutions for evolution equations, Proc. Japan Acad., Ser.A., 67(1991), 323-328.

[CGS] Y.-G. Chen, Y. Giga and K. Sato, On instant extinction for very fast diffusion equations, Discrete and Continuous Dynamical Systems, 3(1997), 243-250.

[CL] M. G. Crandall and P.-L. Lions, Viscosity solutions of Hamilton-Jacobi equations, Trans. Amer. Math. Soc., 277(1983), 1-42.

[CIL] M. G. Crandall, H. Ishii and P.-L. Lions, User's guide to viscosity solutions of second order partial differential equations, Bull. Amer. Math. Soc., 27(1992), 1-67.

[E] L. C. Evans, A geometric interpretation of the heat equations with multivalued initial data, SIAM J. Math. Anal., 27(1996), 932-958.

[ESou] L. C. Evans and P. E. Souganidis, Differential games and representation formulas for solutions of Hamilton-Jacobi equations, Indiana Univ. Math. J., 33(1984), 773-797.

[ESp] L. C. Evans and J. Spruck, Motion of level sets by mean curvature, I. J. Differntial Geometry, 33(1991), 635-681. 
[GG1] M.-H. Giga and Y. Giga, Motion driven by nonlocal curvature in the plane, In: Asymptotics in Nonlinear Diffusive Systems, (eds. Nishiura et al), Tohoku Math. Publ. 8(1998), 75-83.

[GG2] M.-H. Giga and Y. Giga, Generalized motion by nonlocal curvature in the plane, in preparation

[G] Y. Giga, A level set method for surface evolution equations, Sugaku Expositions, 10(1997), 217-241 (translated from Sūgaku 47 (1995), 321-340.)

[GGIS] Y. Giga, S. Goto, H. Ishii and M.-H. Sato, Comparison principle and convexity preserving properties for singular degenerate parabolic equations on unbounded domains, Indiana Univ. Math. J., 40(1991), 443-470.

[GS] Y. Giga and M.-H. Sato, On semicontinuous solutions for general HamiltonJacobi equations, Hokkaido Univ. Preprint Series in Math. \# 459, June (1999).

[I1] H. Ishii, Existence and uniqueness of solutions of Hamilton-Jacobi equations, Funkcialaj Ekvacioj 29(1986), 167-188.

[I2] H. Ishii, Perron's method for Hamilton-Jacobi equations, Duke Math. J., 55 (1987), 369-384.

[IS] H. Ishii and P. E. Souganidis, Generalized motion of noncompact hypersurfaces with velocity having arbitrary growth on the curvature tensor, Tôhoku Math. J., 47(1995), 227-250.

[K] S. Koike, Semicontinuous viscosity solutions for Hamilton-Jacobi equations with a degenerate coefficient, Differential and Integral Equations, 10(1997), 455-472.

[L] P. L. Lions, Generalized Solutions of Hamilton-Jacobi Equations, Research Notes in Math. 69, Pitman, Boston-London-Melbourne, (1982).

[S] A. I. Subbotin, Minimax Inequalities and Hamilton-Jacobi equations, Nauka, Moscow, (1991) (in Russian). 